The Technique.

1. One hour before the administration a hypodermic injection of atropine sulphate $Y_{1}^{1} \sigma$ grain is given with the object of preventing the considerable secretion of mucus in the air passages which may otherwise be caused by ether. This also prevents sweating during the operation, and thereby retains the body heat. Atropine is also a respiratory stimulant and a vagal obtundent, both valuable qualities unhampered by contraindications. Morphine is not advantageous for routine employment, because it appears to induce undue oozing of blood from the wound during operation, and certainly retards the reappearance of the laryngeal reflex and the resumption of consciousness during recovery.

2. When the patient is in position for the ether administration a small mouth prop is inserted between the side teeth and held in position by light pressure upwards upon the lower jaw with the administrator's left hand.

3. A ring pad made of gauze is then rested on the patient's face encircling the nose and mouth.

4. The wire mask fitted with a pad made of sixteen layers of gauze is now laid upon the ring pad, and the patient instructed to breathe in and out slowly through the mouth.

5. The administrator now talks quietly to the patient all the time, while he drops ether continuously upon the mask until unconsciousness supervenes.

6. The mouth is then opened wider with a jaw gag, a tongue clip with finger loop is inserted, and the tongue drawn slightly forward away from the pharynx.

The mouth prop is then readjusted, leaving the mouth partly open as at first. The ring pad and mask are then replaced and the ether drops continued.*

By means of this procedure all further anxiety as to the patency of the airways is removed, and laboured respiration entirely avoided.

\section{Practical Advantages.}

These may be shortly summed up under the following headings :

1. A smooth induction period of six to eight minutes,

2. Very early clouding of the mental faculties.

3. Tranquil regular respiration with abdominal relaxation.

4. Absence of mucus in the air passages.

5. No unusual oozing of blood from the wound.

6. Extraordinary safety, alterations in the patient's position or disturbances of vital structures producing barely any depression.

7. Maintenance of normal blood pressure and absence of shock owing to the obtundent effects of both atropine and ether.

8. Suitability for goître operations and adenoid and tonsil extirpation.

9. The patient may be safely propped upright on pillows on return to bed.

10. No after-effects, vomiting being quite rare.

11. Acidosis improbable.

12. Unusual value therefore in septic conditions and those with flagging circulation.

After four years' employment of open ether administration, in contrast with thirteen years' previous use of other methods, I cannot refrain from strongly urging its general acceptance as a substitute for chloroform and mixtures in all routine surgical work on account of its extreme simplicity, safety, and convenience for the operator, administrator, and patient.

* The mouth props, tongue clip, mask, and sewn gauze pads may be obtained from Hessrs. Allen and Hanburys of Wigmore Street.

THE Lord Mayor, who presided at the annual meeting of the St. Mark's Hospital for Cancer, Fistula, and other Diseases of Rectum, London, E.C., on February 16th, said that his knowledge of the institution was of long standing, since more than fifty years ago he had the pleasure of knowing the founder, and had assisted the staff of the hospital at operations in the early days on a number of occasions. The annual report, moved by Dr. A. W. Oxford, showed that 643 in-patients and 1,997 outpatients had been under treatment during 1911. The addition of six beds increased the cost of provisions and a ialling-off in the income had produced a deficit of $\$ 621$. A vote of thanks to the honorary medical staff, moved by the Lord Mayor, was acknowleged by Mr. Swinford Edwards.

\section{LEFT-SIDED SUBPHRENIC ABSCESS DUE TO PERFORATED DUODENAL ULCER.}

\author{
BY
}

H. D. ROLLESTON, M.D., F.R.C.P., sENIOR PHYSICIAN, ST. GEORGE's HOSPITAL; PHYSICIAN, VICTORIA
HOSPITAL FOR CHILDREN.

This case is remarkable from two points of view, the clinical and the anatomical.

A man aged 66 was admitted on March 1st, 1910, to St. George's Hospital with severe abdominal pain, worse on the right side, and extending from the right costal margin to the iliac fossa. The right half of the abdomen was tender, rigid, distended, and resonant; the liver dullness was diminished. There were signs of a small pleural effusion on the right side. The tongue was dry and coated, and the patient looked pale, ill, and dired and distressed. The temperature was $99^{\circ}$ on admission, the pulse 88 , and the respirations 24 . The urine, 1030, acid, contained indican, gave a slight reduction with Fehling's solution and was free from albumen and pus. He had not been sick, and was not so at any time after admi

Past History.-He has had similar, though less severe, Past History.-He has had similar, though less severe,
attacks: the first was five years ago, and he has had four since, the last sevcn months ago, when he vomited bile and was in bed for three weeks.

Progress of the Case.-The pulse gradually quickened to 118 , the respirations to 36 , and the temperature varied between $97^{\circ}$ and $101^{\circ}$. Definite signs of a gaseous subphrenic abscess on the right side developed; it was noticed that the left loin was dull on percussion, and was oedematous. On March 8th $\mathrm{Mr}$. Dent opened the abdomen by an incision $3 \mathrm{in.} \mathrm{long} \mathrm{through} \mathrm{the} \mathrm{outer}$ side of the right rectus. On opening the peritoneum gas escaped, but there was not any pus. A large mass of thickened escaped there omenturas found extending to the left of the incision beneath the left rectus, and on separating this an abscess containing offensive pus was opened; a large drainage tube was inserted. A few drops of bile were noticed in the discharge; and, though the cause of the abscess was not found, it was thought to be probably a perforated duodenal ulcer. The wound drained freely after wound. The patient gradually sank and died on March 13th, 1910

The necropsy, performed by Dr. Trevor, showed that the gastro-colic omentum was attached by old adhesions to the anterior abdominal walls, from side to side, just below the costal margins. The under surface of the liver was adherent to the stomach, and there were adhesions around the gall bladder. There was a large subphrenic abscess on the left side of the upper abdomen, limited to the right by the left side of the falciform ligament of the liver, and occupying the dome of the stomach chamber. in short, the left anterior intraperitoneal sumarenamber; in short, the left anterior intraperitoneal subphrenic abscess as described by Barnard. A small track passing downwards and to the right under the free margin of the falciform ligament of the liver ran to a perforated duodenal ulcer the size of a shilling on the anterior surface just outside the pyloric ridge. A thin track of pus was found on the convexity of the liver to the right of the falciform ligament, but it did not spread out into an abscess; there were also one or two scattered collections of coagulated pus, the size of a threepenny piece, on the convexity of the right lobe of the liver, but the substance of the liver did not show any suppuration.: As these small purulent areas on the convexity of the right lobe of the liver were quite recent, it is probable that they had formed after the laparotomy.

It is noticeable that in this case the signs pointed to a right-sided gaseous subphrenic abscess, whereas the abscess was really on the left side. In explanation of this want of accord between the physical signs and the real condition, it is highly probable that there was free gas on the right side of the falciform ligament, though the abscess was on the left side. For at the operation air but no pus came out when the peritoneum was opened by the incision to the right of the right rectus muscle.

The main interest of the case is the occurrence of a leftsided subphrenic abscess due to a perforated duodenal ulcer. In this instance the history of past attacks of a similar, though less severe, nature and the presence of adhesions make it highly probable that there had been attacks of local peritonitis in connexion with the duodenal ulcer, and that the adhesions thus produced were responsible for the abscess being on the left side of the abdomen. Left-sided subphrenic abscesses due to perforated duodenal ulcer must be very rare, for the condition is not referred to in Moynihan's work on duodenal ulcer. ${ }^{8}$ From Box's remarks ${ }^{1}$ it appears that a left-sided intraperitoneal abscess might occur even in the absence of adhesions. He says : "The most posterior part of the general peritoneal cavity of the left subphrenic region is situate, when the body is supine, near the posterior border of the spleen, to the outer side of the upper end of the left kidney, just above the 
level of the eleventh intercostal space. It is in this direction that effusions from ruptured gastric or even duodenal ulcers are directed by the slope of the mesial watershed and the presence of the mesocolic shelf." Hunt ${ }^{2}$ records an abscess confined to the lesser sac of the peritoneum with which a duodenal ulcer communicated directly; such an abscess would extend into the left side of the abdomen. REFERENCES.
1 Box, Lancet, Iondon, 1910, i, 851.2 E. R. Hunt, Trans. Med. Soe.
Lond., 1905, xxviii, 76. 3 Moynihan, Duodenat Ulcer, 1910 (W. B.
Saunders Eompeny). Saunders Eompeny).

\section{ON THE TRANSMISSION OF LEPROSY TO ANIMALS BY DIRECT INOCULATION.}

\section{BY H. BAYON, M.D.,}

BEIT MEMORIAL RESEARCH FELLOW.

(From the Lister Institute of Preventive Medicine.)

Apparentuy there exists the widespread opinion that leprosy has never been transmitted to animals by means of direct inoculation of nodules from the human being. In fact, Abraham, in his careful and comprehensive chapter on leprosy in Allbutt and Rolleston's System of Medicine, after reviewing the various attempts to inoculate animals, comes to the conclusion that

The evidence given above, though to some extent conflicting, may on the whole be regarded as supporting the view expressed by Besnier-that leprosy is strictly a human disease which cannot be transmitted to animals.

I need not refer to the similar views held for many years regarding syphilis, which, being a disease due to a protozoon-like germ, might possibly be specific for the human race, as appears to be the case with malaria in man. And yet, after Bertarelli and Parodi succeeded with their first experiments, no one seems to have found it impossible to transmit syphilis to the rabbit.

Leprosy is a disease due to a definite bacterium, and there appears to be no special reason why the rabbit or any other laboratory animal should not be inoculable with it. The existence of spontaneous rat-lepra affords, in fact, the best ground for postulating the possibility of transmitting lepra to animals.

However, in a matter like this it is useless to quibble with words about possibilities. All arguments can be swept aside by the statement of successful inoculation, supported of course by detailed histological evidence.

I am not taking into consideration the infection of animals with leprosy after cultivation of the germ.

Rost has reported that he has produced the clinical features of leprosy in a monkey by injecting his streptothrix.

Duval, by injecting his strain of $B$. leprae in enormous quantities into a monkey, produced lesions which, he says, could not be distinguished from those in the human being. Kedrowsky has produced in mice and rabbits lesions extremely similar to those of human leprosy by injecting the germs he cultivated from lepers. I have had an opportunity of examining his microscopical slides personally, and admit that they are most convincing.

With a diphtheroid-like germ cultivated from a leper I have produced lesions in a mouse indistinguishable from those occurring in spontaneous rat leprosy.

Now, however, I am concerned with reviewing the work done by grafting lepromata to animals direct from the patient. So far as I can gather from the literature on this subject the positive results which have been recorded are as follows :

1881. Neisser, after twenty-four unsuccessful attempts on rabbits, injected two dogs with leprous nodules, and concluded from the results that leprosy developed locally at the site of inoculation.

1883. Damsch grafted-lepromata in the anterior eye chamber of two rabbits. The animals died after 139 and 219 days. Apparently a slight increase in the quantity of acid-fast germs had taken place. He also inoculated two cats in the abdomen with leprous nodules. After 120 days numerous bacilli were found in the newly formed tissue round the atrophied nodules.

1888. Vossius carried out experiments in a similar fashion to Damsch on rabbits and had identical results.
He also considered that the acid-fast germs had multiplied.

1885-6. Melcher and Ortmann inoculated four rabbits in the same way. They succeeded in getting, after four to ten months, definite metastases and deposits of acid-fast germs in the spleen, liver, caecum, pleura, and pericardium. They stained these germs with Baumgarten's differential stain and found that they had the staining properties of Hansen's germ.

1887. Wesener injected several rabbits with leprous nodules, and in two animals out of eight, after periods of four and a half months and eight months respectively, he found lesions apparently identical with those described by Melcher and Ortmann-that is, nodules from the size of a pinhead to that of a pea in the lungs, on the pleura, on the epiploon, in the liver, in the lymphatic glands, spleen, kidneys, caecum, and peritoneal surface. Caseation and giant cells were present. In the eyes of the rabbits he obtained after six and eight months a congregation of round cells choked up with acid-fast germs. . He considered that the generalized lesions were due to tuberculosis and that the ocular lesions were caused by dead bacilli, because acid-fast germs can be demonstrated in the eye of the rabbit after inoculation with nodules which have been kept for years in alcohol.

1901. Barannikow confirmed these results on one rabbit.

1893. Wnoukow inoculated twenty rabbits with leprous nodules in various fashions (intraocularly, subcutaneously, intraperitoneally). In fourteen rabbits he got lesions which he considered to be of tuberculous natare. No confirmatory tests with guinea-pigs appear to have been made.

1893. Tedeschi inoculated a leproma into the dura of a monkey. The animal died after eight days; that is far too brief a period for any conclusions to be drawn.

1902. Iranow injected several guinea-pigs with leprous nodules. In one case the animal was killed after eight months and found to have nodules in the epiploon, which Ivanow. considered were due to multiplication of the bacilli.

1905. Thiroux inoculated five rabbits with leprous material. The animals lived thirteen to twenty months and presented various lesions at autopsy, which Thiroux considered to be tuberculous.

1906. Nicolle inoculated monkeys with ground-up nodules and succeeded in getting localized lesions.

1909. Marchoux and Borret inoculated a chimpanzee under the skin of the ear with a freshly-excised leprous nodule. It apparently increased in size during three months and then began to be resorbed. An examination of the blood showed a few leucocytes with badly-staining bacilli. The animal died ninety-six days after inoculation. The nodule had reached-the size of a split pea, and under the microscope. was shown.to consist of three layers. In the middle were the remains of the original tissue, quite necrosed, with a few loose acid-fast germs. Around this was a considerable number of lymphocytes, enclosing numerons bacilli, and at the periphery organized connective tissue with a few cells filled with bacilli. The authors considered that a proliferation of the original germs had taken place.

1909. Sugai inoculated Japanese dancing mice intraperitoneally with an emulsion of fresh leprous nodules and found as a resuit the development of miliary granulomata on the peritoneal lining, especially. in the hepatic region and epiploon. Also the bronchial and peritoneal glands showed characteristic leprous lesions. All contained acidfast germs. The attempt to transmit the disease from these to other mice failed.

1909. Kitasato appears to have successfully inoculated an orang-outang on the cornea. Only scanty details were, however, given.

1909. Stanziale reports upon some very careful and thorough experiments on rabbits. In one typical case the inoculated into the eye of the animal a fragment of leproma. During the first few days a marked hyperaemia developed round the opening in the cornea. Soon afterwards a constant diminution in the size of the nodule could be made out, accompanied by the formation of an opalescent exudation, which took its departure from the inoculated piece of tissue and spread out as a fan-shaped segment. After twenty days the nodule became stationary 